\title{
Identification of a novel FOXL2 mutation in a single family with both types of blepharophimosis-ptosis-epicanthus inversus syndrome
}

\author{
LIN YANG ${ }^{1,2}$, TUO $\mathrm{LI}^{2}$ and YIQIAO XING ${ }^{1}$ \\ ${ }^{1}$ Department of Ophthalmology, Renmin Hospital of Wuhan University, Wuhan, Hubei 430060; \\ ${ }^{2}$ Department of Ophthalmology, Enshi Medical College of Wuhan University, Central Hospital \\ of Enshi Tujia and Miao Autonomous Prefecture, Enshi, Hubei 445000, P.R. China
}

Received October 9, 2016; Accepted June 8, 2017

DOI: $10.3892 / \mathrm{mmr} .2017 .7226$

\begin{abstract}
Blepharophimosis-ptosis-epicanthus inversus syndrome (BPES) is a rare autosomal dominant disease, which has been divided into two types according to whether it involves premature ovarian failure (POF). Mutations in forkhead box L2 (FOXL2) have been identified in the majority of patients with BPES. The present study aimed to identify the causative mutation in FOXL2 in a Chinese family with both types of BPES. Clinical data and genomic DNA were collected from a single Chinese family with BPES. All the coding exons and adjacent regions of FOXL2 were screened in one affected member to detect the causative mutation using Sanger sequencing. The detected mutation was also screened in available family members and in 100 normal control chromosomes. In total, seven family members were recruited in the present study, including four affected and three unaffected members. The patient (II:5) exhibited typical features of type II BPES, characterized by a narrowed horizontal palpehral aperture, ptosis, epicanthus inversus and telecanthus without POF, whereas the patient's three daughters (III:1, III:2 and III:3) were diagnosed with type I BPES, in which a complex eyelid malformation was accompanied with POF. A novel heterozygous mutation in FOXL2 (c.844_860dup17, p.His291Argfs*71) was found in the four affected members, which was absent in the remaining three unaffected members and in the 100 control chromosomes. This novel duplicate mutation (c.844_860dup17, p.His291Argfs*71) in FOXL2 was identified in a Chinese family with both types of BPES. These
\end{abstract}

Correspondence to: Professor Tuo Li, Department of Ophthalmology, Enshi Medical College of Wuhan University, Central Hospital of Enshi Tujia and Miao Autonomous Prefecture, 154 South Wuyang Road, Enshi, Hubei 445000, P.R. China E-mail: 13986840088@139.com

Key words: forkhead box L2, mutation, blepharophimosisptosis-epicanthus inversus syndrome, premature ovarian failure, Sanger sequence findings expand current knowledge of the mutation spectrum of the FOXL2 gene and confirmed the intra-family phenotypic heterogeneity of BPES.

\section{Introduction}

Blepharophimosis-ptosis-epicanthus inversus syndrome (BPES; OMIM\# 110100) is a rare autosomal dominant condition with a prevalence of $\sim 1 / 50,000$ worldwide (1). It is characterized by typical eyelid malformations, including a narrowed horizontal palpehral aperture, ptosis, epicanthus inversus and telecanthus, and can occur as an isolated type (type II) or accompanied with ovarian dysfunction leading to premature ovarian failure (POF), known as type I BPES (2). Mutations in forkhead box L2 (FOXL2; OMIM 605597) have been found in $88 \%$ of patients with Type I and II BPES (3). Previous studies $(4,5)$ identified BPES families with intraand interfamilial phenotypic variability caused by the same FOXL2 mutation. However, the cause of this variability by the same mutation remains to be fully elucidated. The identification of additional mutations, which lead to the phenotypic variability of BPES, may contribute to revealing their potential pathogenic mechanism to a degree.

The present study reported on a Chinese family with both types of BPES. The study aimed to identify the causative mutation in FOXL2 in this family, which may assist in elucidating the pathogenic mechanism leading to phenotypic variability.

\section{Materials and methods}

Patients. A total of seven members of a Chinese family, including four patients with BPES and three unaffected members, were recruited in the present study. All patients were examined at the Central Hospital of Enshi Autonomous Prefecture (Hubei, China). The diagnostic criteria of BPES included blepharophimosis, ptosis, epicanthus inversus and telecanthus. The diagnostic criteria of type I BPES comprised BPES accompanied with premature ovarian failure (POF), which was defined as amenorrhea for a duration of $\geq 6$ months at the age of $<40$ years and a concentration of follicle-stimulating hormone (FSH) of $>40$ IU/l. Written informed consent was 
A
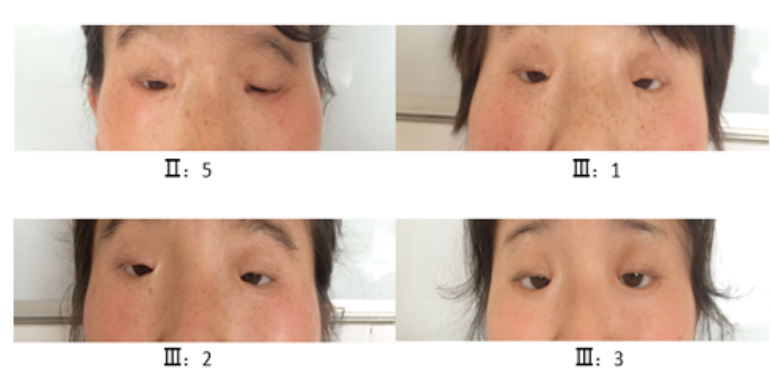

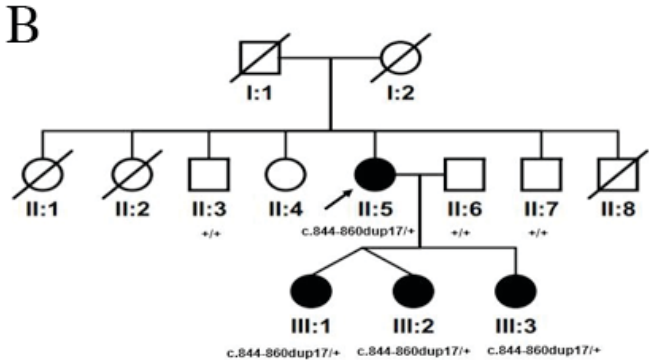

NG_012454.1c.844_860dup17

$\mathrm{C}$ mutated allele CCGGCCGCACCCCCGCCTCCGCCGCACCCCCGCCTCCGCCGCACCCCCACCCGCAT

Normal allele
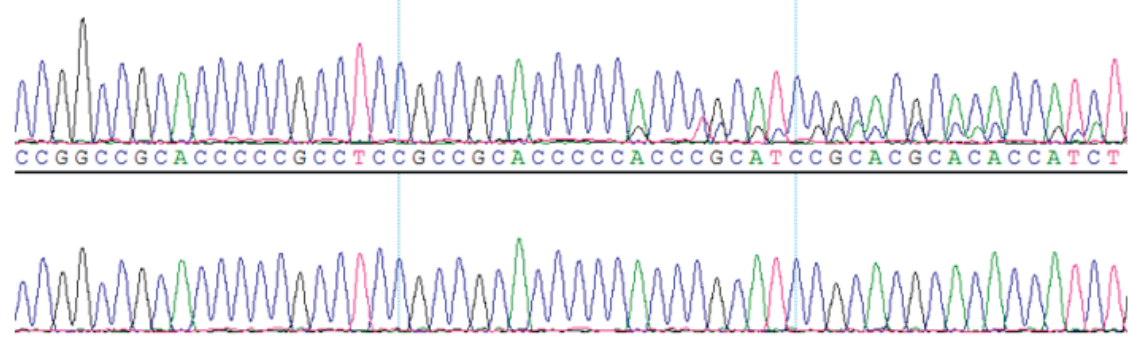

$\mathrm{D}$

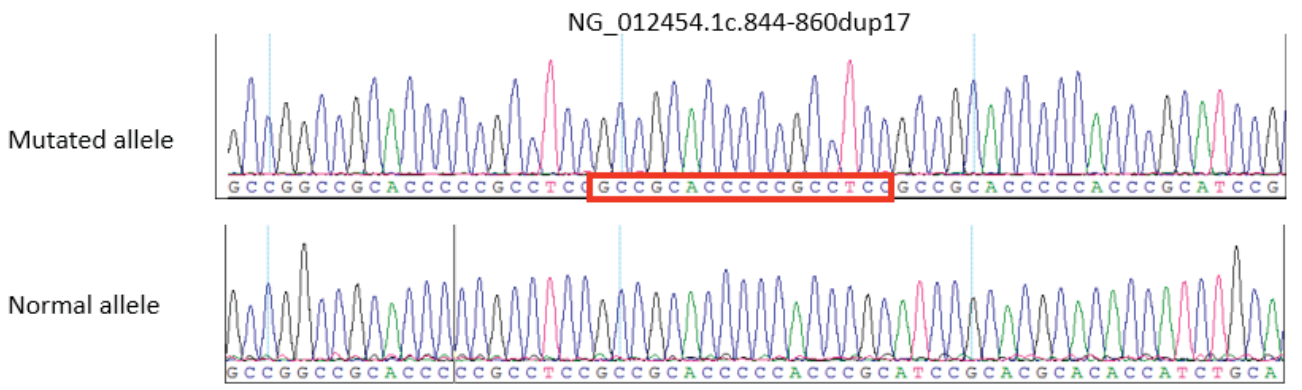

Figure 1. (A) Facial images of patients with BPES from the family examined. Patients exhibited a combination of congenital eyelid anomalies: small palpebral fissures, ptosis, telecanthus and epicanthus inversus. (B) Pedigrees of the family. Squares represent men and circles represent women. Solid symbols represent affected individuals, and their corresponding mutations are shown below the pedigrees (+, wild-type allele). Open symbols represent unaffected individuals The arrow indicates the proband. (C) Sanger sequence chromatography of FOXL2 fragments. The sequence represents the (c.844_860dup17) mutation, and the mutation site is marked with a red box. (D) Cloning sequence chromatography of FOXL2 fragments. The sequence represents the (c.844_860dup17) mutation, and the mutation site is marked with a red box. FOXL2, forkhead box L2.

obtained from every participant according to the guidelines of the Declaration of Helsinki and Guidance of Sample Collection of Human Genetic Disease through the Ministry of Public Health of China. The present study was approved by the ethics committee of the Central Hospital of Enshi Autonomous Prefecture.

Mutation analysis. Genomic DNA was isolated from the peripheral blood using a phenol-chloroform method. Amplification of the entire coding region of FOXL2 was performed using five overlapping sets of primers: forward, TCCGCAGTCTCCAGAAGTTT and reverse, GGCACC TTGATGAAGGACTC, for FOXL2-A; forward, CACAGT CAAGGAGCCAGAAG and reverse, CACGAGTTGTTG AGGAAGCC for FOXL2-B; forward, GAGTGCTTCATC AAG GTGCC and reverse, TTGTACGAGTTCACTACG CC for FOXL2-C; forward, CTTCCTCAACAACTCGTG GC and reverse, TGTCGTGGTCCCAGTAAGAG for FOXL2-D; forward, TCGTACAATGGCCTGGGA G and reverse, ACA AAGAGGAGCGACAGGAG for FOXL2-E. Polymerase chain reaction (PCR) analysis was performed with $64 \mathrm{ng}$ genomic DNA, $12 \mu 1$ 2X GC-rich buffer I (Shanghai bio-engineering, Shanghai, China), $1.6 \mu \mathrm{l}$ of a dNTP mixture ( $2.5 \mathrm{mmol} / \mathrm{l}$, Shanghai bio-engineering, Shanghai, China), 1.5 unit rTaq DNA polymerase (Shanghai bio-engineering, Shanghai, China), $0.5 \mu \mathrm{l}(10 \mu \mathrm{mol} / \mathrm{l})$ of each primer and $\mathrm{ddH}_{2} \mathrm{O}$ to a final volume of $20 \mu \mathrm{l}$. The PCR amplification procedure was performed as follows: Initial step at $95^{\circ} \mathrm{C}$ for $5 \mathrm{~min}$, followed by $15 \mathrm{cycles}$ at $95^{\circ} \mathrm{C}$ for $30 \mathrm{sec}, 65^{\circ} \mathrm{C}$ for $30 \mathrm{sec}$ and $72^{\circ} \mathrm{C}$ for $40 \mathrm{sec}$, and then 21 cycles at $95^{\circ} \mathrm{C}$ for $30 \mathrm{sec}, 58^{\circ} \mathrm{C}$ for $30 \mathrm{sec}$ and $72^{\circ} \mathrm{C}$ for $40 \mathrm{sec}$, followed by a final elongation step at $72^{\circ} \mathrm{C}$ for $10 \mathrm{~min}$. The PCR products were analyzed on an ABI 3130 genetic analyzer (Applied Biosystem, Foster city, CA) with a BigDye Terminator cycle sequencing kit version 3.1. The sequencing results were aligned with consensus sequences from the Genome Bioinformatics database (http://genome.ucsc.edu/) to identify variants using the Seqman II program of the DNASTAR Lasergene package (Lasergene version 7.1; DNASTAR, Madison, WI, USA). Each PCR fragment was confirmed 
Table I. Clinical features of four patients within the family.

\begin{tabular}{|c|c|c|c|c|c|c|c|c|}
\hline \multirow[b]{2}{*}{ Clinical feature (mm) } & \multicolumn{2}{|c|}{ II:5 } & \multicolumn{2}{|c|}{ III: 1} & \multicolumn{2}{|c|}{ III:2 } & \multicolumn{2}{|c|}{ III:3 } \\
\hline & $\mathrm{R}$ & $\mathrm{L}$ & $\mathrm{R}$ & $\mathrm{L}$ & $\mathrm{R}$ & $\mathrm{L}$ & $\mathrm{R}$ & $\mathrm{L}$ \\
\hline Horizontal length of lid & 21 & 21 & 18 & 19 & 20 & 20 & 23 & 23 \\
\hline Vertical length of lid & 3 & 3 & 4 & 4 & 5 & 5 & 6 & 6 \\
\hline Inner canthus interval & \multicolumn{2}{|c|}{$\begin{array}{l}40 \\
82\end{array}$} & \multicolumn{2}{|c|}{40} & \multicolumn{2}{|c|}{$\begin{array}{l}44 \\
86\end{array}$} & \multicolumn{2}{|c|}{35} \\
\hline Outer canthus interval & \multicolumn{2}{|c|}{82} & \multicolumn{2}{|c|}{80} & \multicolumn{2}{|c|}{86} & \multicolumn{2}{|c|}{83} \\
\hline Axis oculi & 24.60 & 23.24 & 27.64 & 29.12 & 24.82 & 24.05 & 29.52 & 30.17 \\
\hline
\end{tabular}

R, right; L, left; II:5, proband; III:1-3, daughters of proband.

Table II. Gynecological examination and clinical details of female patients.

\begin{tabular}{|c|c|c|c|c|}
\hline Feature & II:5 & III:1 & III:2 & III:3 \\
\hline Age (years) & 54 & 32 & 32 & 29 \\
\hline Menarche age (years) & 19 & 16 & 16 & 15 \\
\hline Menstrual cycle & Irregular & Irregular & Irregular & Irregular \\
\hline Menopause age (years) & 49 & Not occurred & Not occurred & Not occurred \\
\hline Pregnancies (n) & 3 & 0 & 0 & 0 \\
\hline FSH (IU/l) & Absence & 38.15 & 15.22 & 10.78 \\
\hline LH (IU/l) & Absence & 22.70 & 24.37 & 12.67 \\
\hline Estradiol $(\mathrm{pg} / \mathrm{ml})$ & Absence & 10.00 & 56.00 & 50.00 \\
\hline Gynecological history & - & Ovarian hypoplasia & $\begin{array}{l}\text { Ovarian hypoplasia; } \\
\text { endometrial hyperplasia }\end{array}$ & $\begin{array}{l}\text { Ovarian hypoplasia; } \\
\text { endometrial hyperplasia }\end{array}$ \\
\hline
\end{tabular}

FSH, follicle-stimulating hormone; LH, luteinizing hormone; II:5, proband; III:1-3, daughters of proband.

by bidirectional sequencing. The variants were further analyzed by cloning sequencing, in which the sequence of the cloned fragment was identified by cycle sequencing as described above. Segregation analysis was performed in all living affected members and available unaffected members in the family. Variants were also screened in 100 control chromosomes. Mutation nomenclature was based on the Human Genomic Variation Society (http://www.hgvs .org/mutnomen/) nomenclature guidelines.

\section{Results}

BPES was inherited in a dominant autosomal pattern in the family investigated (Fig. 1A and B). The female patient (II:5) exhibited typical features of BPES II, characterized by a narrowed horizontal palpehral aperture, ptosis, epicanthus inversus and telecanthus without POF, myasthenia of upper lid and age-related cataract in her left eye. The onset of menopause was at 49 years old, which was substantially older than the age of 40 for POF. The daughters of patient II:5 were diagnosed with type I BPES, in which a complex eyelid malformation (Table I; Fig. 1A) was accompanied with POF. The patients with POF underwent a clinical assessment, which included a relative gynecologic examination and recording of gynecological history (Table II). The mother (patient II:5) had three daughters (III:1, III:2 and III:3), of which III:1 and III:2 were fraternal twins, and all of which were diagnosed with BPES with irregular menstrual cycles and no history of pregnancy in 10 years of marriage. The ovary was not visible in daughter III:1 on transvaginal ultrasonography examination, and a significant increase in FSH and decrease in estradiol were recorded (Table II). Daughters III:2 and III:3 suffered from endometrial hyperplasia, however, this was more serious in the latter, who was diagnosed with endometrial atypical hyperplasia. This showed potential to progress to endometrial cancer and was treated by uterine curettage four times. The three had suffered from amenorrhea for a duration of $\geq 6$ months. The sequence of the cloned PCR products obtained from these patients revealed a novel heterozygous 17-bp duplication mutation, c.844_860dup17 (Fig. 1C and D). This mutation resulted in a truncated protein (p.His291Argfs*71). This mutation was absent in the unaffected family members and in the 100 normal control chromosomes.

\section{Discussion}

In the present study, the novel FOXL2 heterozygous mutation (c.844_860dup17), resulting in a truncated protein 
(p.His291Argfs*71) was identified in a Chinese family in which both types of BPES were present. This was in agreement with previously reported mutational hotspots $(5,6)$. FOXL2 is a single-exon gene, which belongs to the family of winged-helix/forkhead transcription factors (7). It encodes a protein of 376 amino acids, which contains a 110 amino acid DNA-binding forkhead domain and a 14 residue polyalanine (poly-Ala) tract, which is highly conserved among mammals (8-11). To date, $>200$ mutations in FOXL2 have been found to be associated with BPES in different populations, as detailed in the human FOXL2 mutation database (http://medgen.ugent.be/foxl2) (12,13). The proportions of each type are as follows: $11 \%$ missense mutations; $12 \%$ nonsense mutations; $44 \%$ frame-shift mutations; and 33\% in-frame changes (4-6). Previous studies have reported the existence of two mutational hotspots: Mutations leading to an expansion of the poly-Ala tract account for $31 \%$ and novel out-of-frame duplications account for $13 \%$ of all intragenic FOXL2 mutations $(5,6)$. Furthermore, a preliminary genotype-phenotype correlation has been confirmed: Mutations resulting in a predicted truncated protein before the poly-Ala tract may lead to type I BPES, whereas predicted proteins with a poly-Ala tract expansion may cause type II BPES $(4,5)$.

In the present study, a novel heterozygous mutation, c.844_860dup17, which was a 17-bp duplication, was detected. This mutation results in a truncated protein (p.His291Argfs*71), with complete forkhead and ploy-Ala domains. Of note, the novel c.844_860dup17 mutation resulted in type I and type II BPES in a single family. This is not the first report of the same mutation in FOXL2 leading to both types of BPES. De Baere et al (5) described a nonsense mutation c.822C $>\mathrm{G}$ (p.Y274X) in a family, in which the mother was diagnosed with type II BPES, whereas her daughter was diagnosed with type I BPES. Another mutation (c.804-805insC) $(5,14)$ has also been reported to cause both types of BPES in two families. All three of these mutations have been predicted to result in a mutant protein with complete forkhead and ploy-Ala domains. However, the molecular mechanisms underlying the actions of this type of mutation in humans, and its effects on ovarian function and follicle development, remain to be fully elucidated. The results of the present study contribute to the elucidation of genotype-phenotype correlation and further investigations are warranted. Phenotype variability is important for genetic counseling, in order for individuals considering becoming parents to know how severely affected a child is likely to be.

In conclusion, the present study identified a novel mutation (c.844_860dup17) in a Chinese family in which both types of BPES were present. These results expand on the spectrum of FOXL2 mutations and provide confirmation of intra-family phenotypic variations.

\section{Acknowledgements}

The authors would like to thank Qingjiong Zhang of the Department of Ophthalmic Genetics and Molecular Biology, Zhongshan Ophthalmic Center, Sun Yat-sen University (Guangzhou, China) for their guidance. This study was supported by Hubei Province's Outstanding Medical Academic Leader Program, the National Nature Science Foundation of China (grant no. 81,360,154) and the Open Project of the State Key Laboratory of Ophthalmology (grant no. 2013KF04).

\section{References}

1. Oley C and Baraitser M: Blepharophimosis, ptosis, epicanthus inversus syndrome (BPES syndrome). J Med Genet 25: 47-51, 1988.

2. Zlotogora J, Sagi M and Cohen T: The blepharophimosis, ptosis, and epicanthus inversus syndrome:Delineation of two types. Am J Hum Genet 35: 1020-1027, 1983.

3. Crisponi L, Deiana M, Loi A, Chiappe F, Uda M, Amati P, Bisceglia L, Zelante L, Nagaraja R, Porcu S, et al: The putative forkhead transcription factor FOXL2 is mutated in blepharophimosis/ptosis/epicanthus inversus syndrome. Nat Genet 27: 159-166, 2001.

4. De BaereE,Dixon MJ,Small KW,Jabs EW,Leroy BP,DevriendtK, Gillerot Y, Mortier G, Meire F, Van Maldergem L, et al: Spectrum of FOXL2 gene mutations in blepharophimosis-ptosis-epicanthus inversus (BPES) families demonstrates a genotype-phenotype correlation. Hum Mol Genet 10: 1591-1600, 2001.

5. De Baere E, Beysen D, Oley C, Lorenz B, Cocquet J, De Sutter P, Devriendt K, Dixon M, Fryns JP, Garza A, et al: FOXL2 and BPES: Mutational hotspots, phenotypic variability, and revision of the genotype-phenotype correlation. Am J Hum Genet 72: 478-487, 2003.

6. Beysen D, De Jaegere S, Amor D, Bouchard P, Christin-Maitre S, Fellous M, Touraine P, Grix AW, Hennekam R, Meire F, et al: Identification of 34 novel and 56 known FOXL2 mutations in patients with blepharophimosis syndrome. Hum Mutat 29: E205-E219, 2008.

7. Carlsson P and Mahlapuu M: Forkhead tianscription factors: Key players in development and metabolism. Dev Biol 250: 1-23, 2002.

8. Cocquet J, Pailhoux E, Jaubert F, Servel N, Xia X, Pannetier M, De Baere E, Messiaen L, Cotinot C, Fellous M and Veitia RA: Evolution and expression of FOXL2. J Med Genet 39: 916-921, 2002.

9. Cocquet J, De Baere E, Gareil M, Pannetier M, Xia X, Fellous M and Veitia RA: Structure, evolution and expression of the FOXL2 transcription unit. Cytogenet Genome Res 101: 206-211, 2003.

10. Benayoun BA, Caburet S and Veitia RA: Forkhead transcription factors: Key players in health and disease. Trends Genet 27: 224-232, 2011.

11. Lehmann OJ, Sowden JC, Carlsson P, Jordan $\mathrm{T}$ and Bhattacharya SS: Fox's in development and disease. Trends Genet 19: 339-344, 2003.

12. Beysen D, De Paepe A and De Baere E: FOXL2 mutations and genomic rearrangements in BPES. Hum Mutat 30: 158-169, 2009.

13. Beysen D, Vandesompele J, Messiaen L, De Paepe A and De Baere E: The human FOXL2 mutation database. Hum Mutat 24: 189-193, 2004.

14. Kosaki K, Ogata T, Kosaki R, Sato S and Matsuo N: A novel mutation in the FOXL2 gene in a patient with blepharophimosis syndrome: Differential role of the polyalanine tract in the development of the ovary and the eyelid. Ophthalmic Genet 23: 43-47, 2002. 